\title{
Brownian molecular motors driven by rotation-translation coupling
}

\author{
Brian Geislinger and Ryoichi Kawa* * \\ Department of Physics, University of Alabama at Birmingham, Birmingham, AL 35294
}

\begin{abstract}
We investigated three models of Brownian motors which convert rotational diffusion into directed translational motion by switching on and off a potential. In the first model a spatially asymmetric potential generates directed translational motion by rectifying rotational diffusion. It behaves much like a conventional flashing ratchet. The second model utilizes both rotational diffusion and drift to generate translational motion without spatial asymmetry in the potential. This second model can be driven by a combination of a Brownian motor mechanism (diffusion driven) or by powerstroke (drift driven) depending on the chosen parameters. In the third model, elements of both the Brownian motor and powerstroke mechanisms are combined by switching between three distinct states. Relevance of the model to biological motor proteins is discussed.
\end{abstract}

PACS numbers: 87.16.Nn, 87.17.Aa, 05.40.Jc

\section{INTRODUCTION}

The mechanisms of motor protein motility have been a major research topic in biophysics and biomedical research for several decades [1]. Pioneering work by Huxley and his co-workers [2, 3] established a mathematical model for muscle fiber contractions based on a so-called powerstroke [1, 4] involving the conformational change induced by ATP hydrolysis in the cross-bridges formed between the myosin and actin filaments. This conformational change in the neck region of myosin has been confirmed by various experiments [5, 6, 7, 8, 9, 10]. Based on Huxley's model, it has been believed that this conformational change induces a swinging motion of the myosin (lever arm hypothesis) [1, 11], rotational motion about the neck which is in turn converted into linear translational motion. This model suggests that a motor protein utilizing a powerstroke would behave like a stepping motor with a fixed step length per ATP consumption. In addition, the step length is expected to be proportional to the neck length of the proteins.

Recent single molecule experiments testing a variety of protein mutants with different neck lengths support the lever arm mechanism of protein motility 12, 13, 14, 15, 16, 17, 18]. Other recent experiments have emphasized both the length of the motor protein neck as well as the magnitude of the angle through which it swings during a powerstroke in an attempt to explain the unusually long step length of Myosin Id [19]. However, some similar experiments showing unexpected step sizes for a given neck length appear to be inconsistent with the lever arm model 20, 21, 22, 23, 24, 25, 26, 27, 28, 29]. In these experiments, proteins must somehow move over several steps during a single chemical cycle in order to produce the observed step size. Some experiments showed that the speed of the motor protein is independent of the neck length in contradiction with other experiments 23]. Fur-

*Electronic address: kawai@uab.edu thermore, the step length of certain motor proteins seems too long compared with their neck length 30, 31 and some diffusive steps appeared to be necessary [11, 32, 33].

The filaments along which motor proteins move are polymers made up of polar units with a "plus" and a "minus" end. Most myosin-based motor proteins move preferentially towards the plus end of the actin filament track. Myosin VI moves in the opposite direction 27, 28, 31, 34]. In addition, other motor proteins such as Myosin IXb recently have been observed to move in the opposite direction under certain conditions 35, 36]. These anomalous motor proteins are structurally very similar to the their plus-ended counterparts. It has been observed that when the lever arm is engineered in a certain way, the motor moved in the opposite direction [37]. In the case of Myosin VI, it has been speculated that the naturally occurring protein moves preferentially towards the minus end of the filament through a similar mechanism [34]. However, the actual mechanism determining the directionality still remains unknown.

The majority of motor proteins are comprised of two motor domains that work in conjunction with each other to produce directed motion. The two heads are particularly crucial to the models put forth to describe processive motors such as Myosin V, which move through many ATP hydrolysis cycles before detaching from its filament [1, 38]. Most of the prevailing models on processive motor movement require the two motor domains to work in sequence, alternating attachment states allowing the motor to move forward without completely releasing from the filament [33, 39, 40, 41]. However, some motor proteins such as Myosin IXb have only a single motor domain and yet is still a processive motor [35, 36, 42]. In addition, numerous other experiments have been performed on single headed molecular motors, both naturally occurring and derived single-headed mutants. They show a continued ability to move and produce a force against an external load despite the obvious structural deficiencies with regards to the models based on two-head mechanisms [30, 43, 44, 45, 46].

Apart from these recent experimental findings, new models for motor proteins based on the idea of Brown- 
ian motors have been intensively investigated during the last decade 47, 48, 49, 50, 51]. When Brownian particles are in a periodic but spatially asymmetric potential and away from thermal equilibrium, they can generate mechanical work by rectifying thermal fluctuation [52]. Since motor proteins move along an asymmetric filament and ATP hydrolysis creates nonequilibrium conditions, it has been proposed that motor proteins could be described by a Brownian motor model [47, 48, 49, 50, 51]. A Brownian motor based on the flashing ratchet model moves as a step motor in consistent with experiments. However, Brownian motors also provide for the possibility of moving more than one step per chemical cycle in contrast to the powerstroke model. Furthermore, the step length of the Brownian motor is independent on the neck length. These features possibly account for some of the experimental data which the powerstroke model fails to explain 30, 53.

The powerstroke model and Brownian motor model has been thought of as two opposing extremes in the search for motility mechanisms of molecular motors. In the former, thermal fluctuations have no role at all. On the other hand, the latter is incapable of producing motion without thermal fluctuations. As a result, it was commonly believed that only one of them is the correct mechanism. However, it has recently been postulated that both mechanisms may be involved in the actual motor proteins [1, 32].

The purpose of the present work is to develop a single mathematical model that includes both powerstroke and Brownian motor mechanisms. Since the coupling between the rotational and linear translational degrees of freedom is essential to the powerstroke mechanism, our first step is to develop a model which couples rotational motion to linear motion (Section II). In the first model (Section III), we demonstrate that free thermal diffusion in the rotational degree of freedom is sufficient to induce directed linear motion via a flashing ratchet mechanism. A model based on the behavior of motor proteins that is discussed in Section [V] shows directed translational motion driven by either a Brownian motor or a powerstroke mechanism depending on the parameters used. A more realistic motor protein model involving three chemical states is proposed in section $\nabla$ This motor is simultaneously capable of both powerstroke and Brownian motor mechanisms during a single chemical cycle. Relevance of the present models to actual biological motors is discussed in the final section.

\section{ROTATION-TRANSLATION COUPLING}

While various mathematical models for molecular motors driven by rotation-translation coupling have been proposed in the past [54, 55], these models are not closely related to known biological molecular motors. A more realistic model is necessary to explain the experimentally observed behaviors of biological molecular motors, such (a)

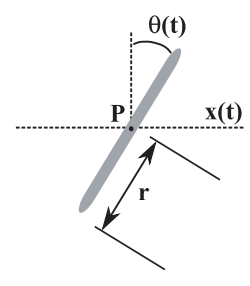

$\theta$

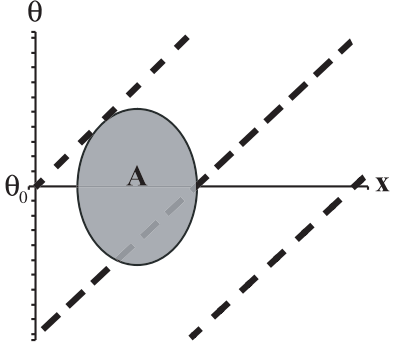

(b)
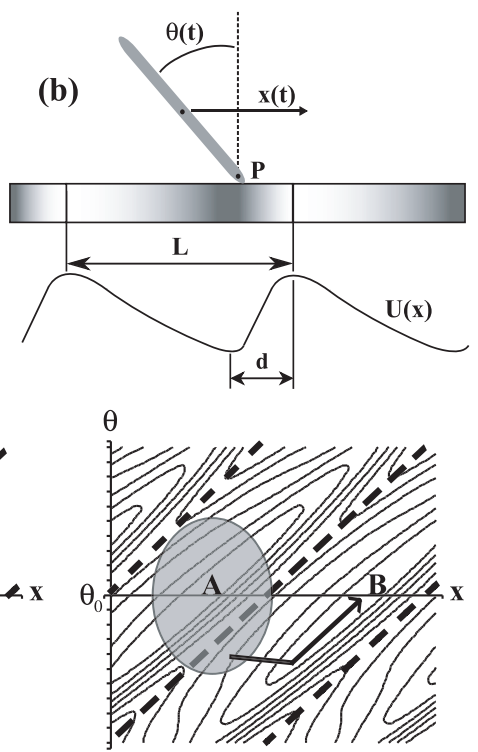

FIG. 1: Model for molecular motors with a coupled rotational and translational mechanism for producing directed motion. (a) Unbound state. Unattached of its filament track, the motor is free to thermally diffuse in both $x$ and $\theta$. The resulting probability distribution $A$ is shown in the lower image. In principle, $D_{x} \neq D_{\theta}$ so $A$ is depicted as an ellipse. The dashed lines depict the location of the potential barriers in the bound state for a point of reference. (b) Bound state. Upon binding to its filament, the pivot point $P$ of the motor shifts towards the binding site, activating the coupling between rotation and translation. The potential contours shown in the lower image corresponding to the bound state, depict the probability of remaining in the same potential well at $A$ or moving to the next at $B$. As can be seen here, an asymmetric potential $U(x)$ is required to bias the motion of the motor.

as step size distributions and velocity-force relationships. Here, we try to develop a mathematically simple, yet sufficiently realistic model by taking into account experimental observations as much as possible.

Motor proteins undergo two major conformational changes during a chemical cycle, first when ATP hydrolysis takes place and second when ADP is released from the protein. The main change occurs between the head and neck of the protein such that the angle between the neck with respect to the head changes. For simplicity, we consider a simple rod of length $\ell=2 r$ as a motor particle whose degrees of freedom are defined as the position of the center of mass $x$ and a rotational angle $\theta$ around the center (measured from the normal to the filament). Since free rotation does not generate translational motion, it is therefore necessary to couple the rotational motion with the translational degree of freedom. Since the release of ADP occurs when the protein is attached to the filament, a large conformational change in the neck associated with the release would produce a rotation about the point of attachment to the filament. The resulting motion about that pivot point produces translational motion of the cen- 
ter of the motor that is proportional to $r \sin \theta$ as shown in Fig. 1]

The potential energy of the motor in the bound state is determined by the location of the contact point $x_{P}$ and the orientation of the motor $\theta$. In general, these two degrees of freedom are weakly coupled but not in a way to produce a translational motion out of the swing motion. Therefore, we assume an uncoupled form of potential energy:

$$
V\left(x_{P}, \theta\right)=U_{\text {rot }}(\theta)+U_{\text {trans }}\left(x_{P}\right)
$$

where $U_{\text {rot }}$ represents a conformational change in the neck region of the protein and $U_{\text {trans }}$ is a binding energy between the motor and the filament. Since the filament is periodic, the binding energy is also periodic as $U_{\text {trans }}\left(x_{P}+L\right)=U_{\text {trans }}\left(x_{P}\right)$ where $L$ is the period of the filament. Noting that $x_{p}$ and center-of-mass coordinate $x$ are related by $x_{P}=x-r \sin \theta \approx x-r \theta$ [56], the binding energy as a function of $x$ and $\theta$ is given by

$$
V(x, \theta)=U_{\text {rot }}(\theta)+U_{\text {trans }}(x-r \theta)
$$

This potential energy function provides the desirable form of coupling between rotational and translational degrees of freedom, allowing for the conversion of rotational motion to translational motion as shown below.

\section{A TWO-STATE BROWNIAN RATCHET DRIVEN BY ROTATIONAL DIFFUSION}

Although motor proteins in general have several chemical states during ATP hydrolysis, we will restrict this discussion to a two-state model describing a bound and unbound state for a motor protein. When a potential is absent (unbound state) the motor freely diffuses in its two degrees of freedom, $x$ and $\theta$. As soon as the potential is turned on (bound state), the motor drifts toward a local potential minimum. Switching between these two states will produce net movement in the $x$ direction when the potential is asymmetric along $x$. To illustrate this ratchet mechanism, consider motors confined to an equilibrium point $A$ of the potential well, as seen in Fig. 1(b). When the potential is removed, the motors diffuse over the shaded area in Fig. 1(a). As the potential turns on again, a portion of the shaded area now belongs to the basin of next potential minimum $B$ in Fig. 11 b). The motors that reached this area advance to the new minimum $B$. The rest of the motors move back to the original minimum $A$. In the particular case shown in Fig. 1] the translational diffusion alone is too small to generate directed motion. However, the addition of the rotational diffusion brings the motors to the next basin.

This two-dimensional Brownian ratchet mechanism can be put into a simple mathematical expression in a slow switching limit [48]. Assuming that the potential $U_{\text {trans }}$ is sufficiently deep and the transitions between unbound and bound states are slow, the initial probability distribution of the motors is given by $P(x, \theta, t=0)=$
$\delta\left(x-x_{A}\right) \delta\left(\theta-\theta_{0}\right)$. When the potential is removed, the distribution spreads with diffusion constants $D_{x}$ and $D_{\theta}$ as

$$
\begin{aligned}
& P(x, \theta, t)=P_{\text {rot }}(\theta, t) P_{\text {trans }}(x, t) \\
& =\frac{1}{2 \pi t \sqrt{D_{x} D_{\theta}}} \exp \left(-\frac{\left(x-x_{a}\right)^{2}}{2 D_{x} t}-\frac{\left(\theta-\theta_{0}\right)^{2}}{2 D_{\theta} t}\right) .
\end{aligned}
$$

When the potential is reinstated at $t=\tau$, the probability that the motors leave the basin of the current potential wells in the forward $(+\mathrm{x})$ and backward $(-\mathrm{x})$ direction are given by

$$
\begin{aligned}
W_{+}(\tau) & =\int_{-\infty}^{\infty} d x \int_{-\infty}^{(x-d) / r} d \theta P(x, \theta, \tau) \\
& =\frac{1}{2} \operatorname{erfc}\left(\frac{d}{\sqrt{\left(2 D_{x}+2 r^{2} D_{\theta}\right) \tau}}\right)
\end{aligned}
$$

and

$$
\begin{aligned}
W_{-}(\tau) & =\int_{-\infty}^{\infty} d x \int_{-(x+L-d) / r}^{+\infty} d \theta P(x, \theta, \tau) \\
& =\frac{1}{2} \operatorname{erfc}\left(\frac{L-d}{\sqrt{\left(2 D_{x}+2 r^{2} D_{\theta}\right) \tau}}\right),
\end{aligned}
$$

where $d$ is the shortest distance between the potential minimum and maximum, [see Fig. 1(b)] and erfc is the complementary error function. If the motor is in the unbound state for period $t_{u}$ and in the bound state for $t_{b}$ during one on-off cycle, then the average velocity of the motor is about

$$
\bar{v} \approx \frac{L}{t_{b}+t_{u}}\left(W_{+}\left(t_{u}\right)-W_{-}\left(t_{u}\right)\right) .
$$

Here we neglected the possibility for the motors to diffuse beyond the nearest neighbor potential wells. When the potential is symmetric $(d=L / 2), W_{+}=W_{-}$and thus no net directed motion occurs.

The above discussion can be further confirmed by a direct simulation of Langevin equations for the overdamped motor operated at a temperature $T$ :

$$
\begin{aligned}
& \gamma_{x} \frac{d x}{d t}=-\frac{\partial V(x, \theta)}{\partial x} \sigma(t)+\sqrt{2 k_{B} T \gamma_{x}} \xi_{x}(t)+F \\
& \gamma_{\theta} \frac{d \theta}{d t}=-\frac{\partial V(x, \theta)}{\partial \theta} \sigma(t)+\sqrt{2 k_{B} T \gamma_{\theta}} \xi_{\theta}(t),
\end{aligned}
$$

where $F$ is an external load and $\xi_{i}$ is a Gaussian white noise define by

$$
\left\langle\xi_{i}(t) \xi_{j}\left(t^{\prime}\right)\right\rangle=\delta_{i j} \delta\left(t-t^{\prime}\right) \quad i, j \in(x, \theta) .
$$

The on-off function $\sigma(t)$ takes values 0 and 1 alternatively with transition rates $k_{o n}$ and $k_{o f f}$. Although in general $\gamma_{x}$ would have an angular dependency according to the current orientation of the rod, here we assume that it takes constant values. 


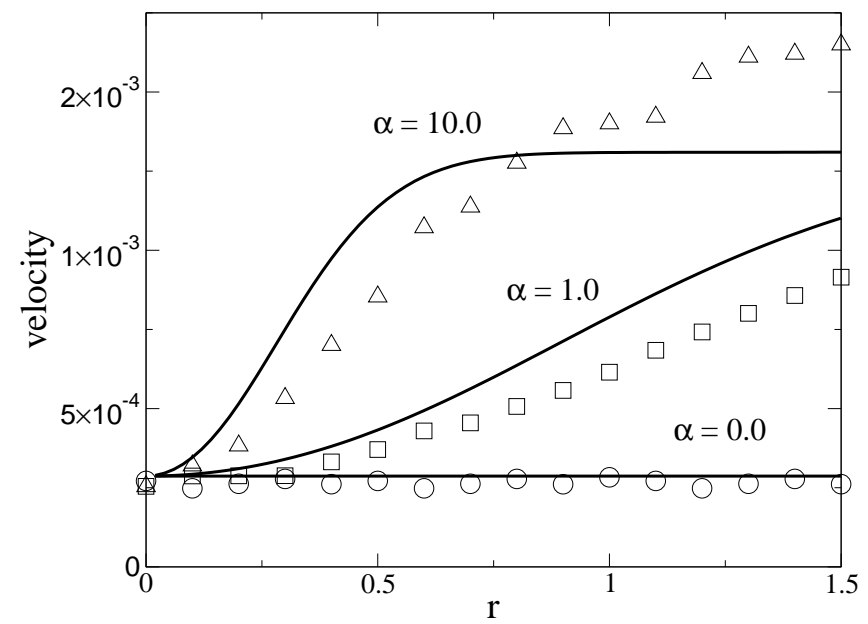

FIG. 2: The relationship between average motor velocity and the motor radius $r$. The circle markers correspond to $\alpha=0.0$, the squares to $\alpha=1.0$, and the triangles to $\alpha=10.0$. The case $\alpha=0.0$ corresponds to a purely translational ratchet. For nonzero $\alpha$ with a sufficiently large value for $r$, the rotational component of the motion provides a unique contribution to the motor velocity. The solid lines correspond to the long time limit velocity obtained from Eq. 6] Parameter values: $t_{u}=5.0, t_{b}=100.0, K=1.0, D=0.0075, d=0.36$.

As a simple model system we use a ratchet potential in the $x$ direction:

$$
U_{\text {trans }}(x)=U_{0}\left[\frac{3}{4} \cos \left(\frac{2 \pi}{L} x\right)-\frac{1}{4} \sin \left(\frac{4 \pi}{L} x\right)\right] .
$$

and a simple harmonic potential with a spring constant $K$ for rotation:

$$
U_{\text {rot }}(\theta)=\frac{K}{2}\left(\theta-\theta_{0}\right)^{2} .
$$

For simplicity, a value of $K=1.0$ will be used throughout the current study.

To reduce the number of parameters, we normalize time, distance and energy as

$$
\tilde{t} \equiv \frac{U_{0} t}{L^{2} \gamma_{x}}, \quad \tilde{x} \equiv \frac{x}{L}, \quad \tilde{U} \equiv \frac{U}{U_{0}}
$$

and accordingly parameters are normalized as $\tilde{r}=r / L$, $\tilde{K}=K / U_{0}, \tilde{F}=F L / U_{0}$. For simplicity, we will omit the tilde on the normalized quantities. Then, the equations of motion (7) become

$$
\begin{aligned}
& \frac{d x}{d t}=-\frac{\partial V(x, \theta)}{\partial x} \sigma(t)+\sqrt{2 D} \xi_{x}+F \\
& \frac{d \theta}{d t}=-\alpha \frac{\partial V(x, \theta)}{\partial \theta} \sigma(t)+\sqrt{2 \alpha D} \xi_{\theta}
\end{aligned}
$$

where the diffusion constant is defined by $D=k_{B} T / U_{0}$. A dimensionless constant $\alpha=r^{2} \gamma_{x} / \gamma_{\theta}$ is determined by the detailed shape of the motor. The contribution of rotational degree of freedom is controlled by the shape of

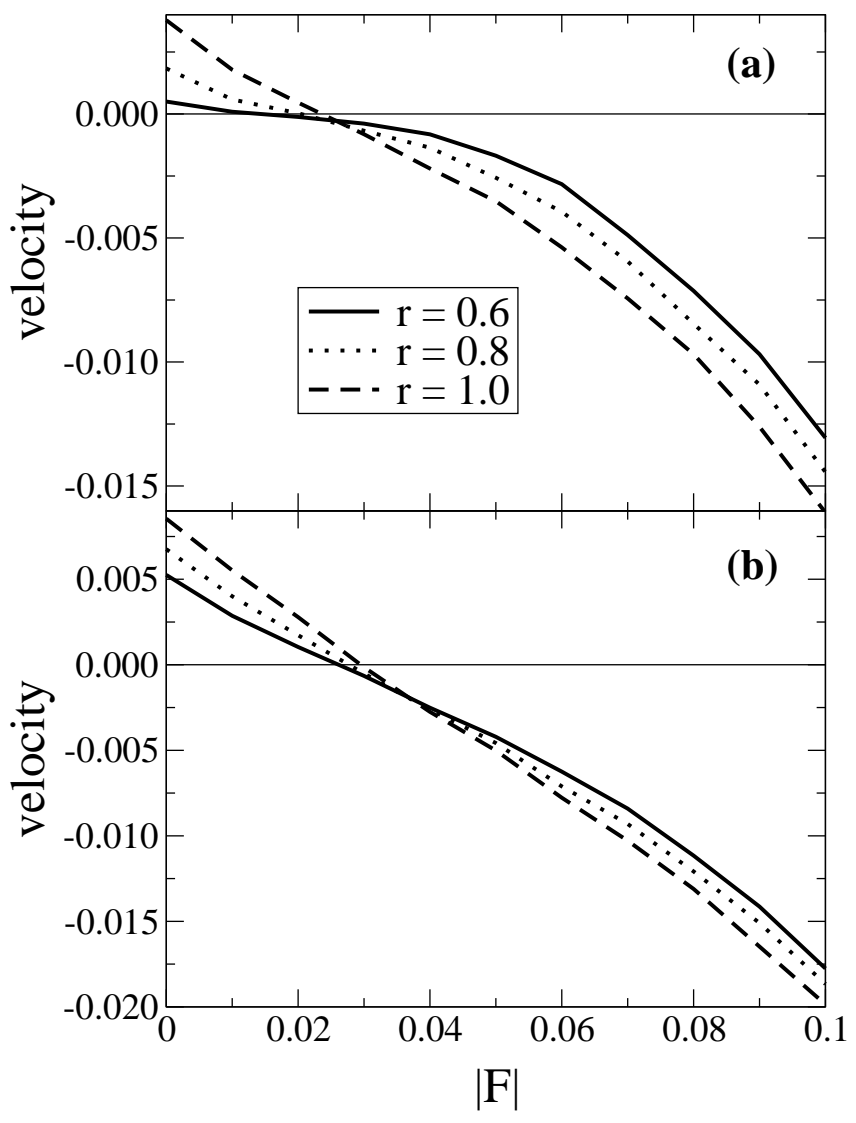

FIG. 3: The effect of applying a constant load $F<0$ to a motor according to the equations of motion given by Eq. (12). (a) We see a distinct nonlinear behavior towards the application of an increasing external load $F$ when the motion relies primarily upon the rotational ratchet mechanism. Parameter values: $\alpha=10.0 ; K=1.0 ; k_{o n}=k_{o f f}=0.5$. (b) The nonlinear behavior disappears when equal proportions of both translational and rotational ratchet are used. Parameter values: $\alpha=1.0 ; K=1.0 ; k_{o n}=k_{o f f}=0.5$.

the motor and hence the value of $\alpha$. When $\alpha=0$, the rotational motion has no contribution and the present model is equivalent to a conventional flashing ratchet. For $\alpha>1$, the rotational diffusion plays a more significant role than the translational diffusion.

Integrating the coupled Langevin equations in Eq. (12) with the Heun method, we can explore the dynamics of this stochastic model. Examining the trajectories of numerous motors allows us to determine the average behavior of the system. For our purposes, an average over 500 realizations of the system will be sufficiently accurate.

Figure 2 shows that the velocity contains no dependence on $r$ for $\alpha=0.0$ as expected. For nonzero values of $\alpha$, the velocity begins to increase with the motor length $r$ beyond the values expected from the translational ratchet alone, showing that the rotational component of the motion introduces an additional mechanism for producing directed motion in the motor. The speed of the motor increases in a roughly linear fashion with respect to the 
motor length $r$ similar to the way that most motor proteins are thought to behave. As the length of the motor increases, a change in the angle $\theta$ will give the motor a longer reach, enabling it to move several potential periods over one cycle with a sufficiently long motor. As seen in Fig. 22 the rotational motion contributes to the motion with increasing $r$ until the motor is long enough to reach the nearest neighboring potential well. Beyond a certain length, the velocity is expected to saturate, until it is then long enough to reach the next nearest neighbor. This behavior is corroborated by the expected behavior of the long time limit case for these various values of $\alpha$. Figure 2 shows close agreement between Eq. 6 described above and the stochastic simulations.

By applying a constant load $F<0$, we can investigate the ability of this model to do work against a load. Figure 3 shows the results of two cases: $\alpha=10.0$ where the rotational diffusion dominates and $\alpha=1.0$ where rotational and translational diffusion equally contribute to the motion. The limit where the motor moves primarily under rotational diffusion exhibits a nonlinear response to an applied load. Upon moving towards the limit of equal contributions (i.e. $\alpha=1.0$ ) the nonlinearity disappears. The nonlinearity is therefore due to the anisotropic diffusion.

\section{A TWO-STATE BROWNIAN MOTOR WITH POWERSTROKE}

In the previous model, coupling between the rotational and translational degree of freedom takes place only when the motor protein is bound to an active site on the filament. The rotation toward an equilibrium angle advances the motor to a minimum within the basin of the potential well. We shall call this rotation the forward stroke. The forward stroke however cannot drive the motor over the potential barrier and only diffusion, rotational or translational, brings the motor to the next potential well. On the contrary, in the standard powerstroke model conformational change of the motor protein drives the motor without diffusion. The key point of the powerstroke model is the presence of the recovery stroke which the motor performs when it is unbound. Molecular motors typically consist of a motor domain attached to a neck and tail region. The tail region typically binds to some sort of cellular cargo or, in the case of skeletal muscle, the tail binds together with other proteins to form a bundle that works in tandem. As opposed to our free floating motor presented in the previous section, the motor protein does not freely rotate in the unbound state and instead is driven to an equilibrium angle by the recovery stroke.

As an alternate model to our previous Brownian ratchet, we consider a motor which is not entirely free to move, but is tethered by one end to some external object assumed to be much larger than itself. When the motor is unbound, it rotates about the pivot point $P^{\prime}$
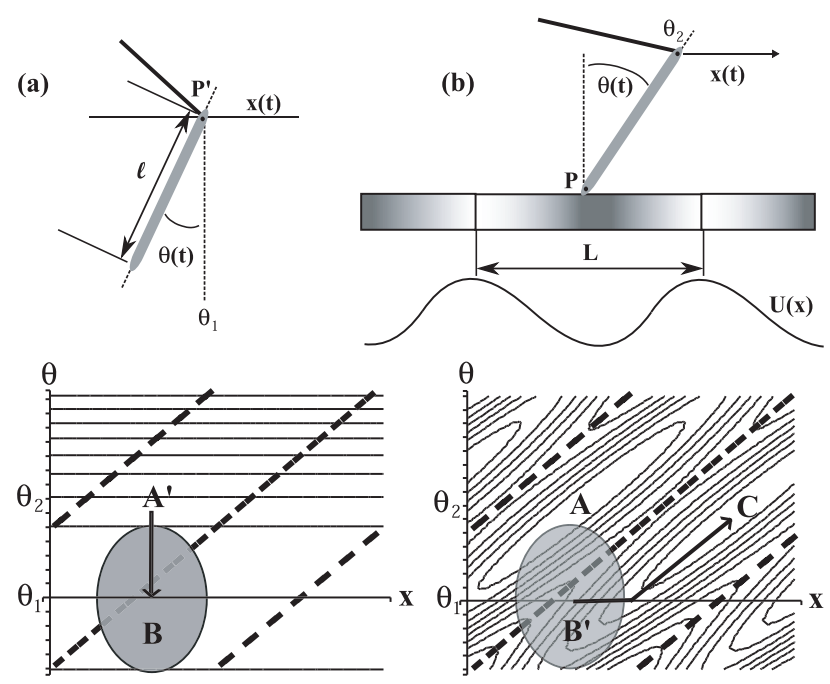

FIG. 4: A model for motor proteins with one end of a motor of length $\ell$ tethered to an external cargo. (a) Unbound state. As before, the motor is free to diffuse in $x$ and $\theta$, but $\theta$ drifts toward a stable angle $\theta_{1}$. Overlaid on the potential contours is the new probability distribution at $B$ centered about $\theta_{1}$, away from the potential minimum of the bound state (shown at $A^{\prime}$ ). (b) Bound state. On binding, the pivot $P$ shifts, giving rise to the coupling term in $U_{\text {trans }}(x)$. Here the stable angle for the bound state $\theta_{2}$ is not necessarily equal to $\theta_{1}$. Given a large enough change in the protein conformation between the bound and unbound states $\ell \Delta \theta=\ell\left(\theta_{2}-\theta_{1}\right)$, motion can occur deterministically without the need for the probability distribution at $B$.

toward an equilibrium angle $\theta_{1}$ (recovery stroke) as il-

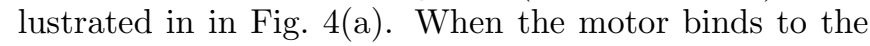
filament, the pivot point shifts to the other end of the motor, $P$ in Fig. U(b). The motor rotates to a new equilibrium angle $\theta_{2}$ about this point (forward stroke). The two different equilibrium angles $\theta_{1}$ and $\theta_{2}$ represent conformational changes in the motor proteins due to ATP hydrolysis.

Suppose that the motor is located at a bound state $A$ in Fig. 世(b). Upon detaching from the filament, the recovery stroke takes the motor from $A^{\prime}$ to $B$ in Fig. 4 (a). If the conformational change, $\ell \Delta \theta=\ell\left(\theta_{2}-\theta_{1}\right)$ is large enough, the point $B$ is in the basin of the next potential well. When it binds to the filament, the forward stroke bring the motor to $C$, generating a net translational motion from $A$ to $C$. This process is entirely deterministic and the directed motion is generated simply by alternating forward and recovery rotations without diffusion. However, the motor is still subject to large thermal fluctuation, which may hamper the powerstroke. Indeed, the thermal fluctuation brings some motors back to the basin

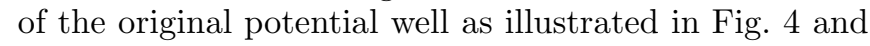
thus reduces the average speed. On the other hand, the thermal fluctuations also make a positive contribution to the directed motion especially when the powerstroke fails. For example, one could imagine a motor prema- 
turely binding to the filament before the recovery stroke completes. In a deterministic model that powerstroke would fail to advance the motor to the next potential well. With the addition of the thermal diffusion, some of the motors that would fail the powerstroke are able to reach the next potential well with help of that diffusion. Furthermore, when the conformational change $\ell \Delta \theta$ is too small to reach the next minimum, the Brownian motion is still capable of producing directed motion on average.

Unlike the previous model, rotational diffusion in the unbound state is accompanied by a drift due to the conformational change $\ell \Delta \theta$. This rotational drift determines the direction of motion. When the sign of $\ell \Delta \theta$ is reversed, the motor moves in the opposite direction. Recent experiments have exhibited such flux reversal for motors modified to swing their neck in the opposite direction to the original motors [34, 37]. Since the symmetry is broken by these conformational changes, it is not necessary for the potential to break the symmetry along the $x$ axis. A simple periodic potential in the normalized units described earlier will suffice for our purposes:

$$
U_{\text {trans }}(x)=\cos (2 \pi x) .
$$

The net potential for the unbound and bound states are respectively given by

$$
\begin{aligned}
& V_{1}(x, \theta)=\frac{K}{2}\left(\theta-\theta_{1}\right)^{2} \\
& V_{2}(x, \theta)=\frac{K}{2}\left(\theta-\theta_{2}\right)^{2}+U_{\text {trans }}(x-\ell \theta),
\end{aligned}
$$

where $x$ is now the position defined by the neck of the motor protein at $P^{\prime}$. The movement of the motors is determined by the Langevin equations in normalized units:

$$
\begin{aligned}
& \frac{d x}{d t}=-\frac{\partial V(x, \theta, t)}{\partial x}+\sqrt{2 D} \xi_{x}+F \\
& \frac{d \theta}{d t}=-\alpha \frac{\partial V(x, \theta, t)}{\partial \theta}+\sqrt{2 \alpha D} \xi_{\theta}
\end{aligned}
$$

where the time-dependent potential is defined as $V(x, \theta, t)=V_{1}(x, \theta)[1-\sigma(t)]+V_{2}(x, \theta) \sigma(t)$.

We begin by examining the relative change in the conformation $\ell \Delta \theta$ between the two states $V_{1}$ and $V_{2}$. Figure 15 shows the average velocity of the motor as $\ell \Delta \theta$ is increased for several different values of the diffusion constant $D$. In the case of $D=0.0$, the thermal fluctuations are disabled. For a small neck length $\ell$ or a small $\Delta \theta$, no velocity is observed. However, once the critical value of $\ell \Delta \theta \approx 0.5$ is reached, the motor begins to move without the need of the thermal fluctuations. This deterministic motion corresponds to a powerstoke motion, related both to the neck length of the motor given by $\ell$ and the angle through which the motor rotates between the unbound and bound states. Although in principle the motor moves forward one potential through each hydrolysis cycle, close to the critical value of $\ell \Delta \theta$ the motor would require a very long switching time to relax to the
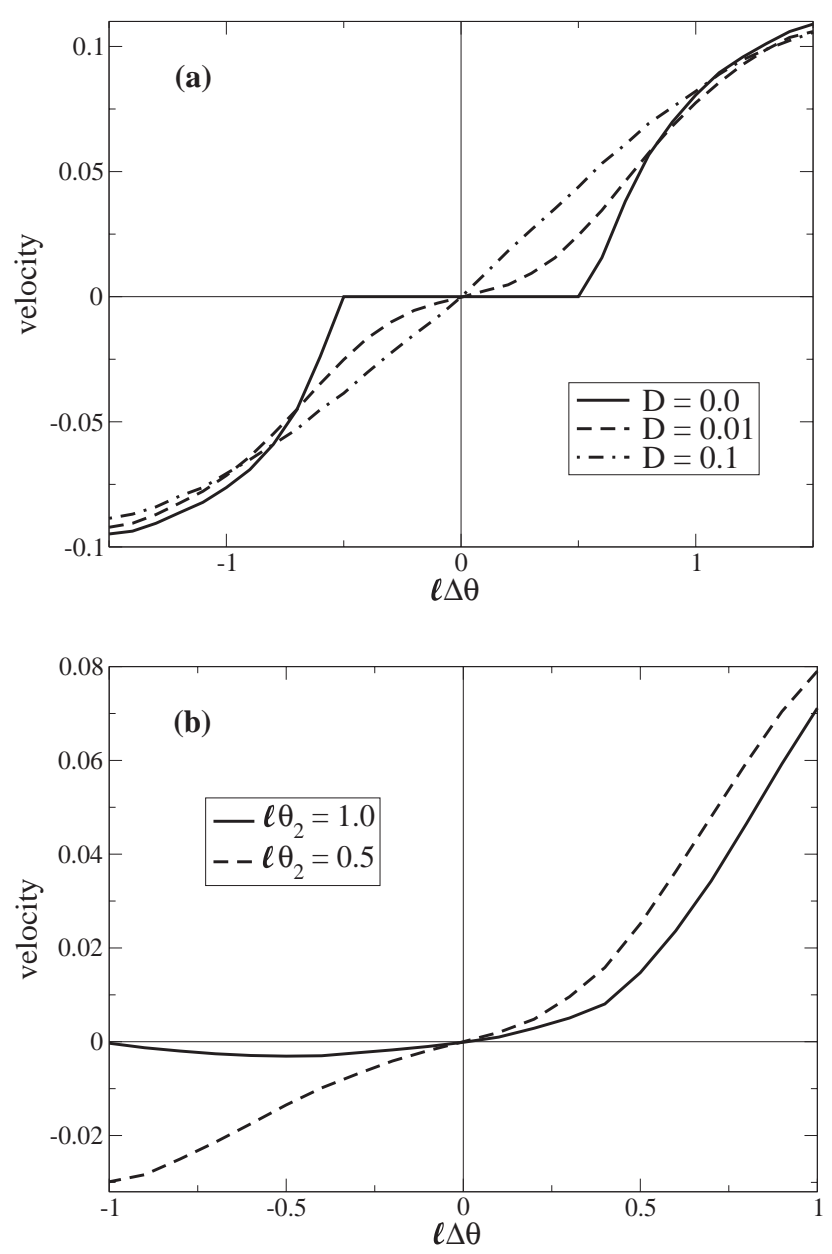

FIG. 5: (a) The average velocity of the motor $\langle\dot{x}\rangle$ as a function of the change in the stable conformations $\ell \Delta \theta$ between the two states with the unbound angle $\theta_{1}$ fixed. Parameter values: $t_{u}=t_{b}=4.0 ; K=1.0 ; \theta_{1}=0.0 ; \alpha=1.0$. (b) The average velocity as a function of $\Delta \theta$ with the bound stable angle $\theta_{2}$ fixed. Parameter values: $t_{u}=t_{b}=4.0 ; K=1.0 ; D=0.01 ; \alpha=1.0$.

potential minimum of the bound state in order to achieve that expected velocity.

In the case of $D \neq 0$, Fig. [5 shows that even for small values of $D$ motion can be seen in the region of $\ell \Delta \theta<0.5$ where the deterministic powerstroke was not possible. The thermal fluctuations of the medium surrounding the motor can be rectified to produce a nonzero average velocity. The mechanism for rectifying the thermal fluctuation follows the Brownian ratchet mechanism from the coupled rotational and translational coupling described in Section III

Also of interest is the case where $\ell \Delta \theta<0$. This parameter regime would be equivalent to a motor taking a power stroke in a negative direction, and as a results Fig. [5 shows an average negative velocity for the motor. This observation is of potential interest in describing the motion of motor proteins such as Myosin VI, observed to 

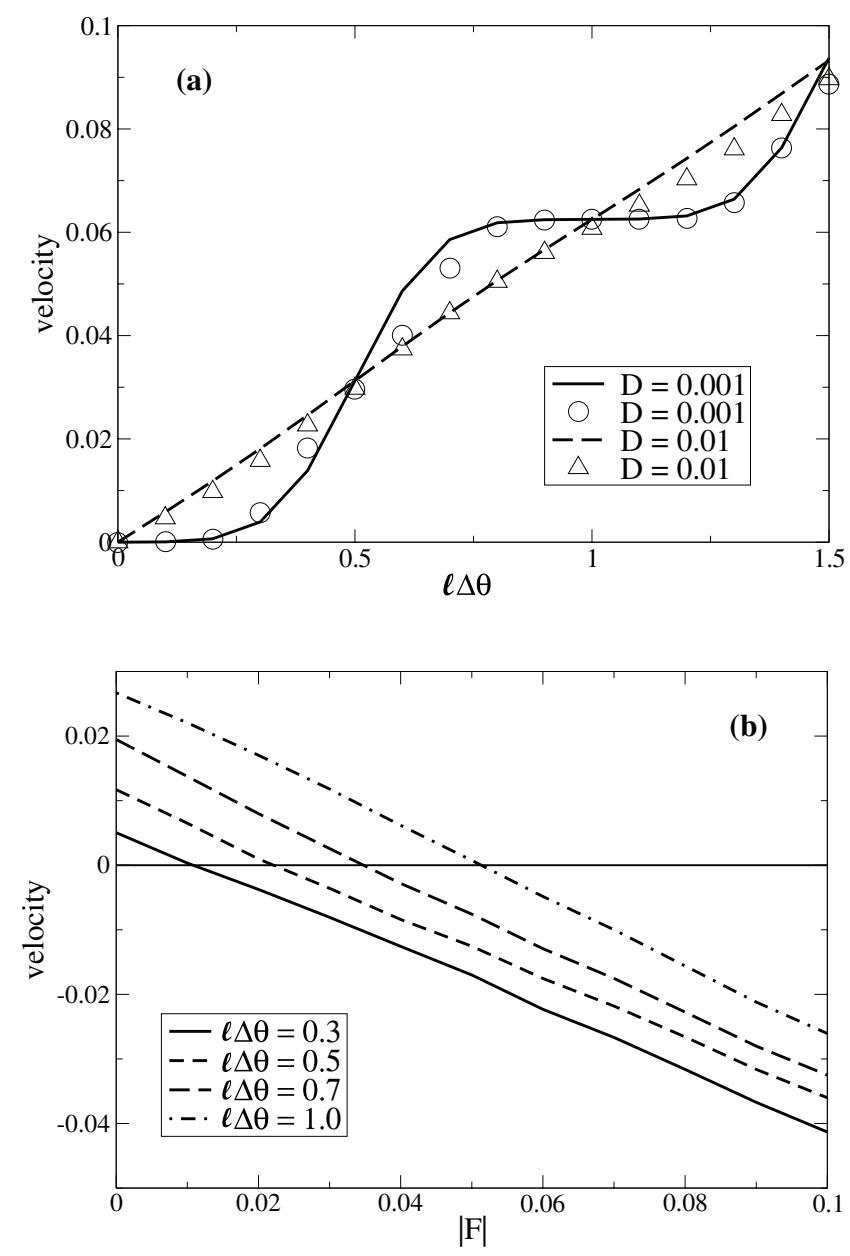

FIG. 6: (a) An analysis of the tether motor system showing both the case of small and large values of $D$. The markers correspond to the Langevin simulation and the lines correspond to the long time limit theory. Parameter values: $t_{u}=t_{b}=16.0$; $K=1.0 ; \alpha=1.0$. (b) An external load $F<0$ applied to a 2 state tethered motor system. Parameter values: $t_{u}=t_{b}=16.0$; $K=1.0 ; D=0.01 ; \alpha=1.0$.

move in the opposite direction as the plus-ended Myosin $\mathrm{V}$ proteins. Using the angle $\theta_{1}=0.0$ for the unbound configuration gives a completely symmetric response given the symmetric nature of $U_{\text {trans }}(x)$. On the other hand, by fixing the bound stable angle $\theta_{2}$ to some non-zero value, the symmetry disappear. To meet the conditions for $\ell \Delta \theta<0$ with $\theta_{2}$ fixed, $\theta_{1}$ must become increasingly large to obtain any negative motion. The harmonic potential term in Eq. (14) quickly becomes too large to sustain negative motion and drives the motor back towards the positive. It is likely that this case is not well represented by the current model and this behavior is an artifact of the approximations that $U_{\text {rot }}$ is a harmonic potenial and $r \sin \theta \approx r \theta$.

In summary, the diffusionless curve in Fig. 5 shows the contribution of the deterministic motion. When the $\ell \Delta \theta$ becomes very large $(\ell \Delta \theta>1.0)$, the deterministic motion dominates the model. For small changes in the angle $(\ell \Delta \theta<0.5)$, the Brownian ratchet mechanism dominates. Between these two limits a combination of the two mechanisms contribute to the average velocity of the motor. The resulting model forms a very robust motor that can produce directed motion under a wide variety of parameters.

As before, the ratcheting motion found in this model can be studied analytically in the long time limit as described before with little difference. However the limits of the integral in Eq. (4) change to take into account the change the angle $\theta_{i}$ between states. The new forms for $W_{+}$and $W_{-}$can be obtained by replacing $d$ with $L / 2-\ell \Delta \theta$ in Eqs. (4) and (5). A nonzero $\ell \Delta \theta$ breaks the symmetry of the system and gives rise to $W_{+} \neq W_{-}$ even though we are using a symmetric potential. The resulting current then follows the same form as stated in Eq. (6). In comparing the results of the long time limit calculation to the stochastic simulations in Fig. 6(a), we again find close agreement to the motion seen in the long time limit.

Motor proteins typically bind a large cargo to their tail in order to transport it from one part of a cell to another. As before, we can apply an external load $F<0$ to these motors to study their ability to pull such a load that might be attached to it. In Fig. [6(b), the same type of linear behavior is observed as before with the previous model. However, Figure 6 (b) also shows that as the value of $\ell \Delta \theta$ is increased the maximum force with which the motor pulls increases. It has been postulated that a Brownian ratchet is unlikely to be a mechanism for motor proteins as those models tend to produce forces too small to account for the forces observed experimentally [1]. Based on the present results, it appears that as we increase the contribution of powerstroke to the function of the motor, the ability of the motor to transport cargo more effectively also increases.

\section{A THREE STATE MODEL}

The cyclic chemical reaction that myosin undergoes as it hydrolyzes ATP can be expressed with varying degrees of precision involving numerous substeps of the reaction [1, 57, 58]. Detailed analysis of the structure of myosin S1 has indicated the existence of at least three distinct conformational states dependent on the state of the bound ATP molecule. [5, [6, 7, 8, 9, [59]. In addition, some myosin working strokes have been resolved into at least two distinct substeps [26, 32, 60], yielding more evidence of multiple conformations of the motor protein. Here, we will utilize three distinct conformational states with a chemical reaction scheme similar to the simple mechanochemical trigger model put forth by Keller and Bustamante [57. This setup enables us to create a hybrid motor that contains elements of both a Brownian motor as well as a deterministic powerstroke. 


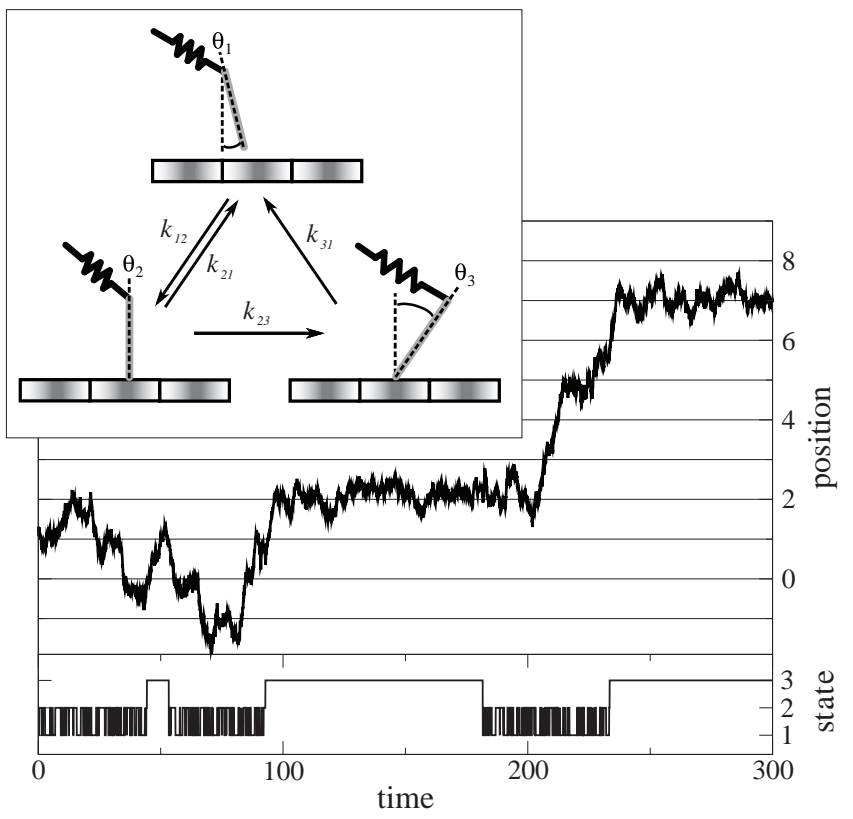

FIG. 7: A three state reaction scheme for a motor protein and a typical trajectory for such a motor. Rate constants governing the reaction are labeled as $k_{i j}$ and the conformational angle for each state is $\theta_{i}$ where $i, j \in(1,2,3)$. Trajectory parameter values: $D=0.01 ; \alpha=1.0 ; k_{12}=0.5, k_{21}=0.2, k_{23}=0.2$, $k_{31}=0.004 ; U_{2}=0.2, U_{3}=1.0 ; K=1.0 ; \ell \Delta \theta_{12}=0.2, \ell \Delta \theta_{23}=1.0$.

Following the cyclic reaction scheme shown in Fig. 7 the transition between a motor containing ATP in an unbound state and its subsequent weakly bound state to an actin filament as the ATP is broken down into ADP and $\mathrm{P}_{i}$ is reversible. This transition induces a small conformational change $\ell \Delta \theta_{12}=\ell\left(\theta_{2}-\theta_{1}\right)$ which is not big enough to perform a powerstroke. However a back and forth transition between this unbound and weakly bound state can generate directed motion by rectifying rotational diffusion as discussed in the previous sections. The transition from a weakly bound to a strongly bound state involves the irreversible release of the inorganic phosphate $\mathrm{P}_{i}$ and is accompanied by a larger change in the conformational state $\ell \Delta \theta_{23}=\ell\left(\theta_{3}-\theta_{2}\right)$ providing the powerstroke. Completing the cycle involves the release of ADP and binding of a new ATP molecule, returning the motor protein to an unbound state. Cycling along these three states, the motor has the capability of utilizing both the Brownian motor and powerstroke mechanisms.

Comparing the typical trajectory of this model shown in Fig. 7 to single molecule experiments performed in recent years shows a possible mechanism explaining observed behavior. The model presented here exhibits occasional backward steps and multiple forward steps during the Brownian ratchet phase similar to the events observed by Yanagida's group [20, 30]. The additional powerstroke as the motor enters the strongly bound state provides another step forward along the potential, sim-
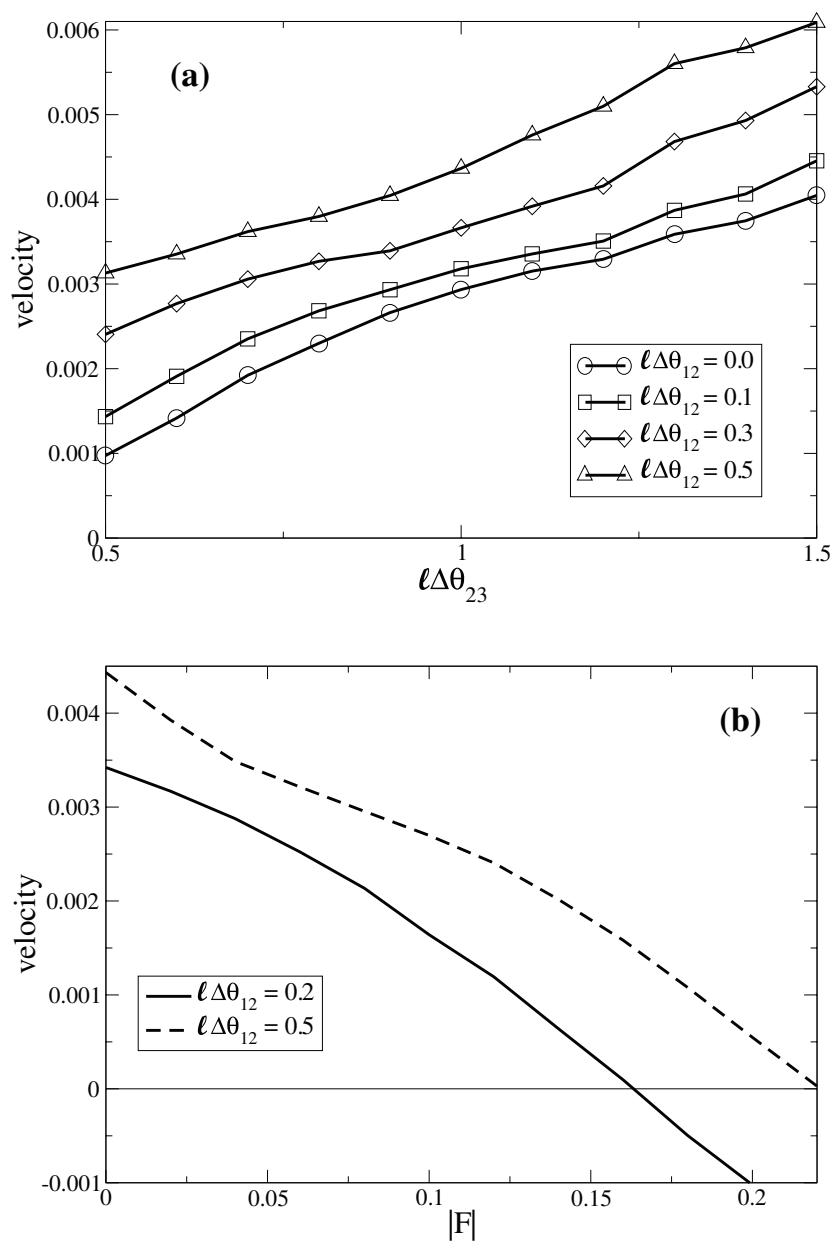

FIG. 8: (a) Motor velocity as a function of varying conformational changes from state 1 to 2 , and from state 2 to 3 . Parameter values: $D=0.01 ; \alpha=1.0 ; k_{12}=0.5, k_{21}=0.2, k_{23}=0.2$, $k_{31}=0.004 ; U_{2}=0.2, U_{3}=1.0 ; K=1.0$. (b) Motor velocity as a function of an applied load $F<0$. Parameter values: as in (a) with $\ell \Delta \theta_{23}=1.0$.

ilar to the two stage working stroke observed in some experiments [26, 32, 60].

Figure 8 (a) demonstrates the change in velocity as both the magnitude of the powerstroke and the conformational change associated with the Brownian motor are increased. In the case of $\ell \Delta \theta_{12}=0$, the Brownian motor mechanism makes no contribution to the velocity of the motor. As $\ell \Delta \theta_{12}$ is increased, the Brownian motor mechanism has the capability of tripling the overall speed of a motor, implying that the motor could take on average two extra steps during the Brownian motor phase of the hydrolysis cycle with adequate tuning of the parameters. As seen in Fig. 8(b), this three state system has the added advantage of withstanding a larger external load opposing the motion than the simpler two state model. 


\section{DISCUSSION}

Conventional Brownian ratchets encounter various difficulties when applied to biological motors. In the present model, coupling the translational and rotational degrees of freedom preserves the conventional translational ratchet mechanism, but also provides a means to produce translational motion through rectifying thermal fluctuations in the rotational degree of freedom. Further, with the addition of an asymmetric conformational change between bound and unbound states, we obtain a model capable of rectifying thermal fluctuations without the need for an asymmetric potential. In the conventional Brownian ratchet model the direction of the motion is determined by a spatial asymmetry in the translational degree of freedom. However, in the present model, the direction of motion is determined by the asymmetry in the conformational change in the motor proteins rather than the asymmetry in the filament, consistent with experimental observation [34, 37]. If this conformational change between the bound and unbound states is large enough, the motor gains the added ability to move by powerstroke without diffusion. Depending on the parameters chosen, either mechanism can be utilized ultimately gaining the benefits of both.

With the minimal three chemical state description detailed in the previous section, we introduce a realistic model for single headed motor proteins, which captures various qualitative properties of motor proteins. Recent experiments on monomeric Myosin I have emphasized the importance of both the neck length and the degree of neck rotation in determining the step size of a motor protein 19]. The present model takes both factors into account in defining the coordinate of conformational change as $\ell \Delta \theta$. Other single molecule experiments have resolved at least two distinct conformational changes during the working stroke of Myosin I [60], one corresponding to a displacement of $\sim 6.5 \mathrm{~nm}$ and another of $\sim 5.5 \mathrm{~nm}$, both very close to the size of the actin filament repeat. The multiple steps observed in Myosin I can be explained by the different phases of motion through an ATPase cycle of the present model. As seen in the trajectory in Fig. [7 a diffusive step followed by a powerstroke would each produce displacement in a series of steps equal to the filament periodicity $L$.

Many single molecule experiments with Myosin II have observed step sizes on the order of $4-10 \mathrm{~nm}$ 20, 30, 61, 62, 63, 64, 65. However, according to Tanaka et al. [63], a random orientation of myosin with respect to an actin filament produces the observed average working stroke of $\sim 5.5 \mathrm{~nm}$. When myosin is close to its natural orientation with respect to actin as it would be in a whole fiber, the step size is closer to three times that value, or $\sim 15 \mathrm{~nm}$. Kitamura et al. [30] observed substeps of $\sim 5.3 \mathrm{~nm}$ along actin subunits. As the dwell time of these substeps had no dependence on ATP concentration, it was proposed that an average of 2.5 substeps during a single ATPase cycle makes up the observed $\sim 13 \mathrm{~nm}$ step per ATP molecule consumed. A simple deterministic motor protein should take a single step per ATP molecule consumed. However, as illustrated in Fig. 8(a), a motor protein with $\ell \Delta \theta_{12}=0.5$ on average should take roughly two 5- $\mathrm{nm}$ diffusive steps along the filament subunits in addition to the powerstroke during the consumption of a single ATP for a total step size of $15 \mathrm{~nm}$.

In the case of dimeric, processive motor proteins like Myosin V, models for the processivity involve some type of coordination between the two motor domains of the myosin. Investigations into single-headed Myosin V motors have revealed a directional force dependence on the kinetics that regulate ATP hydrolysis [45, 46]. These force dependent kinetics indicate a possible method of communication between the two individual motor domains. When the leading head of the motor pair finds its binding site on the actin filament and enters a strongly bound state, the strain caused on the trailing head encourages its release from the filament. At the same time the release of the leading head is slowed, effectively anchoring the motor protein to the filament allowing the trailing head to swing forward and find its next binding site. While this motion of the trailing head forward is thought as a powerstroke process [10, 33, 39, 40, 41], some recent experiments suggest an alternative. The working stroke of a single-headed Myosin V motor protein produces a displacement of only $\sim 25 \mathrm{~nm}$, significantly shorter than the observed step size of $36 \mathrm{~nm}$ for wild type two-headed Myosin V [32]. The motion of this wild type Myosin V has since been resolved into a $12 \mathrm{~nm}$ step followed by a $24 \mathrm{~nm}$ step [66]. Due to the insufficient length of the working stroke of a single-headed Myosin V motor, it has been proposed that the powerstroke of the leading head partially positions the trailing head and the remaining distance is covered by a diffusive process 11, 32]. Others have shown that single headed Myosin V molecules can move processively with the motor protein held in close proximity to the filament 44]. In this study it was proposed that through a series of diffusive steps utilizing a strain sensors similar to the one demonstrated by Veigel et al. [45] and Purcell et al. [46], a single motor protein can achieve a step size of $32 \mathrm{~nm}$.

Myosin VI, another dimeric motor protein, has a neck length much less than that of Myosin V, yet still produces a step length of similar size 26, 31]. As a result, any deterministic powerstroke of this motor protein probably has little to do with its step and is most likely driven by thermal motion. The present model provides a single mechanism allowing a motor to take advantage of both diffusion as well as a subsequent powerstroke/anchoring. Given the large neck of Myosin V, a significant powerstroke on the order of $25 \mathrm{~nm}$ would be reasonable based on a large value for $\ell$, followed by a Brownian motor phase positioning the trailing head at its full step distance from the leading head. On the other hand, a model for Myosin VI could rely heavily upon the Brownian motor mechanism in order to reproduce the observed behavior. We will further explore these dimeric motor proteins 
based on our model in a future paper [67].

Some monomeric motor proteins such as Myosin IX are thought to involve a diffusive step in order to achieve some measure of processivity [35, 42, 68, 69]. However, to maintain this processivity monomeric motor proteins must remain in the vicinity of a filament while in a diffusive state. Both Nalavadi et al. 68] and Kambara et al. 69 have proposed a mechanism. The structure of Myosin IX contains a unique insertion in the motor domain that may harbor a second binding site. This insertion could tether the protein to its filament while still allowing enough freedom for the protein to diffuse. The motor protein then diffuses towards its next binding site before entering another powerstroke. Given the assumption of this special tether in Myosin IX, the present model provides a mechanism to efficiently guide the motor to its next binding site by rectifying thermal fluctuations before entering a strongly bound state.

All of these single molecule constructs have been observed to produce a force on the order of a few $p N$ 1, 44, 45, 46, 61, 64, 70]. Examining the normalized load $F$ from Eq.12), for $L=5.4 \mathrm{~nm}$, the approximate length of an actin subunit, and $U_{0}=20 k_{B} T$ one unit of normalized force is about $15.8 \mathrm{pN}$. The maximum forces generated by the monomers shown in Fig. 8(b) are then approximately $2.6-3.5 p N$, comparable to the forces observed experimentally.

In conclusion, we have developed a realistic model of motor proteins which simultaneously includes the traditional Brownian ratchet model and the powerstroke model in a simple unified form. The properties of this model are qualitatively consistent with recent experimental results. Quantitative investigation is currently underway. Furthermore, we are applying the present model to dimeric processive motors as well as muscle fiber. Although the model is developed for the myosin superfamily, we believe it can be applied to other molecular motors such as kinesin.

\section{ACKNOWLEDGMENTS}

The authors would like to acknowledge Kimmie Farris and Erin Darnell for their help in this work. This work was partially supported by National Science Foundation (NSF) GK-12 Award No. 0139108.
[1] J. Howard, Mechanics of Motor Proteins and the Cytoskeleton (Sinauer Associates, Inc., Sunderland, MA, 2001).

[2] A. F. Huxley, Prog. Biophys. Biophys. Chem. 7, 255 (1957).

[3] A. F. Huxley and R. M. Simmons, Nature 233, 533 (1971).

[4] M. J. Tyska and D. M. Warshaw, Cell Motility and the Cytoskeleton 51, 1 (2002).

[5] I. Rayment, W. R. Rypniewski, K. Schmidt-Base, et al., Science 261, 50 (1993).

[6] M. Whittaker, E. M. Wilson-Kubalek, J. E. Smith, et al., Nature 378, 748 (1995).

[7] J. D. Jontes, E. M. Wilson-Kubalek, and R. A. Milligan, Nature 378, 751 (1995).

[8] A. Houdusse, V. N. Kalabokis, D. Himmel, A. G. SzentGyorgyi, and C. Cohen, Cell 97, 459 (1999).

[9] A. Houdusse, A. G. Szent-Gyorgyi, and C. Cohen, PNAS 97, 11238 (2000).

[10] J. N. Forkey, M. E. Quinlan, M. A. Shaw, J. E. T. Corrie, and Y. E. Goldman, Nature 422, 399 (2003).

[11] M. A. Geeves, Nature 415, 129 (2002).

[12] M. Anson, M. A. Geeves, S. E. Kurzawa, et al., EMBO J. 15, 6069 (1996).

[13] T. Q. P. Uyeda, P. D. Abramson, and J. A. Spudich, PNAS 93, 4459 (1996).

[14] T. Sakamoto, F. Wang, S. Schmitz, et al., J. Bio. Chem. 278, 29201 (2003).

[15] T. J. Purcell, C. Morris, J. A. Spudich, et al., PNAS 99, 14159 (2002).

[16] D. H. Schott, R. N. Collins, and A. Bretscher, J. Cell. Biol. 156, 35 (2002).

[17] C. Ruff, M. Furch, B. Brenner, et al., Nat. Struc. Biol.
8, 226 (2001).

[18] D. M. Warshaw, W. H. Guilford, Y. Freyzon, et al., J. Biol. Chem. 275, 37167 (2000).

[19] D. Köhler, C. Ruff, E. Meyhofer, and M. Bahler, J. Cell Biol. 161, 237 (2003).

[20] A. Ishijima, H. Kojima, T. Funatsu, et al., Cell 92, 161 (1998).

[21] S. Itakura, H. Yamakawa, Y. Y. Toyoshima, et al., Biochem. Biophys. Res. Commun. 196, 1504 (1993).

[22] K. M. Trybus, E. Krementsova, and Y. Freyzon, J. Biol. Chem. 274, 27448 (1999).

[23] C. Perreault-Micale, A. D. Shushan, and L. M. Coluccio, J. Biol. Chem. 275, 21618 (2000).

[24] K. Homma, J. Saito, R. Ikebe, et al., J. Biol. Chem. 275, 34766 (2000).

[25] H. Tanaka, K. Homma, A. H. Iwane, et al., Nature 415, 192 (2002).

[26] I. Lister, S. Schmitz, M. Walker, et al., EMBO J. 23, 1729 (2004).

[27] S. Nishikawa, K. Homma, Y. Komori, M. Iwaki, T. Wazawa, A. Hikikoshi Iwone, J. Saito, R. Ikebe, E. Katayama, T. Yanagida, et al., Biochem. Biophys. Res. Commun. 290, 311 (2002).

[28] R. S. Rock, S. E. Rice, A. L. Wells, T. J. Purcell, J. A. Spudich, and H. L. Sweeney, PNAS 98, 13655 (2001).

[29] A. H. Iwane, H. Tanaka, S. Morimoto, A. Ishijima, and T. Yanagida, Journal of Molecular Biology 353, 213 (2005).

[30] K. Kitamura, M. Tokunaga, A. H. Iwane, et al., Nature 397, 129 (1999).

[31] M. Y. Ali, K. Homma, A. H. Iwane, et al., Biophys. J. 86, 3804 (2004).

[32] C. Veigel, F. Wang, M. L. Bartoo, J. R. Sellers, and J. E. 
Molloy, Nat Cell Biol 4, 59 (2002), ISSN 1465-7392.

[33] R. D. Vale, J. Cell Biol. 163, 445 (2003).

[34] A. L. Wells, A. W. Lin, L.-Q. Chen, D. Safer, S. M. Cain, T. Hasson, B. O. Carragher, R. A. Milligan, and H. L. Sweeney, Nature 401, 505 (1999).

[35] A. Inoue, J. Saito, R. Ikebe, and M. Ikebe, Nat. Cell Biol. 4, 302 (2002).

[36] C. B. O'Connell and M. S. Mooseker, Nat. Cell Biol. 5, 171 (2003).

[37] G. Tsiavaliaris, S. Fujita-Becker, and D. J. Manstein, Nature 427, 558 (2004).

[38] S. Leibler and D. A. Huse, J. Cell Bio. 121, 1357 (1993).

[39] A. Yildiz, J. N. Forkey, S. A. McKinney, et al., Science 300, 2061 (2003).

[40] M. J. Tyska and M. S. Mooseker, Trends Cell Biol. 13, 447 (2003).

[41] A. Vilfan, Biophys. J. 88, 3792 (2005).

[42] P. L. Post, M. J. Tyska, C. B. O'Connel, K. Johung, A. Hayward, and M. S. Mooseker, J. Biol. Chem. 277, 11679 (2002).

[43] T. Yanagida, K. Kitamura, H. Tanaka, A. H. Iwane, and S. Esaki, Curr. Opin. Cell Biol. 12, 20 (2002).

[44] T. M. Watanabe, H. Tanaka, A. H. Iwane, S. MakiYonekura, K. Homma, A. Inoue, R. Ikebe, T. Yanagida, and M. Ikebe, PNAS 101, 9630 (2004).

[45] C. Veigel, S. Schmitz, F. Wang, and J. R. Sellers, Nat Cell Biol 7, 861 (2005).

[46] T. J. Purcell, H. L. Sweeney, and J. A. Spudich, PNAS 102, 13873 (2005).

[47] M. O. Magnasco, Phys. Rev. Lett. 71, 1477 (1993).

[48] R. D. Astumian and M. Bier, Phys Rev Lett 72, 1766 (1994).

[49] R. D. Astumian, Science 276, 917 (1997).

[50] F. Jülicher, A. Ajdari, and J. Prost, Rev. Mod. Phys. 69, 1269 (1997).

[51] F. Jülicher and J. Prost, Phys Rev Lett 75, 2618 (1995).
[52] P. Reimann, Phys. Rep. 361, 57 (2002).

[53] T. P. Terada, M. Sasai, and T. Yomo, PNAS 99, 9202 (2002).

[54] Y.-X. Li, Physica A 251, 382 (1998).

[55] M. Porto, Phys. Rev. E. 63, 030102(R) (2001).

[56] Here we assumed $\theta<<1$ just for symplicity. Although actual angle may not be small, the main conclusions in the present work are valid without this approximation.

[57] D. Keller and C. Bustamante, Biophys. J. 78, 541 (2000).

[58] R. D. Astumian, Appl. Phys. A 75, 193 (2002).

[59] P. D. Coureux, H. L. Sweeney, and A. Houdusse, EMBO J. 23, 4527 (2004).

[60] C. Veigel, L. M. Coluccio, J. D. Jontes, et al., Nature 398, 530 (1999).

[61] J. E. Molloy, J. E. Burns, J. Kendrick-Jones, R. T. Tregear, and D. C. S. White, Nature 378, 209 (1995).

[62] A. D. Mehta, J. T. Finer, and J. A. Spudich, PNAS 94, 7927 (1997).

[63] H. Tanaka, A. Ishijima, M. Honda, K. Saito, and T. Yanagida, Biophys. J. 75, 1886 (1998).

[64] M. J. Tyska, D. E. Dupuis, W. H. Guilford, J. B. Patlak, G. S. Waller, K. M. Trybus, D. M. Warshaw, and S. Lowey, PNAS 96, 4402 (1999).

[65] N. M. Kad, A. S. Rovner, P. M. Fagnant, P. B. Joel, G. G. Kennedy, J. B. Patlak, D. M. Warshaw, and K. M. Trybus, J. Cell Biol. 162, 481 (2003).

[66] S. Uemura, H. Higuchi, A. O. Olivares, E. M. De La Cruz, and S. Ishiwata, Nat. Struc. Mol. Biol. 11, 877 (2004).

[67] B. Geislinger and R. Kawai, in preparation.

[68] V. Nalavadi, M. Nyitrai, C. Bertolini, N. Adamek, M. A. Geeves, and M. Bahler, J. Biol. Chem. 280, 38957 (2005).

[69] T. Kambara and M. Ikebe, J. Biol. Chem. 281, 4949 (2006).

[70] A. D. Mehta, R. S. Rock, M. Rief, J. A. Spudich, M. S. Mooseker, and R. E. Cheney, Nature 400, 590 (1999). 